\title{
A Research on Scheduling Model of Simulated Annealing Algorithm based on Integer Programming
}

\author{
Yaning Yan \\ ZTE Telecommunication College, Xi'an Peihua Universty, Xi'an, 710125, China \\ 394811297@qq.com
}

Keywords: arranging; integer programming; model; annealing algorithm

\begin{abstract}
According to the possession of the school resources, this paper analyzes and presents the key elements and constraints of the course arrangement. Based on this, it establishes an optimized mathematical model of course scheduling determining the objective function and mainly describing the quantitative relations of the Course Arrangement .Meanwhile, the popular method of annealing algorithm is employed to solve the problem to study the scheduling model.
\end{abstract}

\section{Introduction}

Timetabling problem is not only to coordinate various multidimensional conflicts among teaching resources under certain constraints, but also to meet with some soft constrains as far as possible, making it more reasonable and meeting the requirements of human nature.

Integer programming is an integer mathematical programming that requires all or some of the variables are integers. If all the variables are integers, it is called a pure integer programming; if some of the variables are integers, then it is known as mixed integer programming. In mathematical programming, the optimal solution may be a fraction or a decimal, but in some specific issues the result must be an integer. This paper is designed to build a mathematical model of timetable, optimizing the schedule of universities.

\section{The Description of Timetable Problem}

2.1 Analysis on Teaching Schedule. Problems confronted are as follows: (1) the regional problem of course arrangement. The school has Chang a Campus, the main one, and High tech Campus. Course arrangement begins at the same time in the two campus, while most courses are arranged in the Chang an Campus. (2) the problem of major coincidence. There are different schools to open courses in Chang a Campus. Except for students from professional entrance examination, these courses are also for those from adult college entrance examination and self professional, so major coincidence arises. For instance, Computer basic course opens for students from professional entrance examination and those from adult college entrance examination, while its requirements are different. (3) multi grade and multi class problem. There are three-year college students, four- year undergraduate and students who will learn from 3 to 5 years in Adult Education Institute. And majors in each school generally have multiple class, which leads to the complex characteristics of the teaching task, increasing the difficulty of the schedule arrangement; especially for the accounting professional major which has about 20 classes each grade. (4) the concentration of the teachers' time distribution. Our campus is located in remote area, far away from the urban area, teachers need to travel back and forth, if the curriculum arrangement is not appropriate, it will lead to overload running for the teachers in the urban areas and schools, increasing travel times or long time staying in the Chang'an campus. (5) the importance of curriculum and the choice of time. Based on the learning rules and habits of students, the time of course should be based on the difficulty and the importance of curriculum arrangement, if the arrangement is not appropriate, it will lead to poor learning effect. (6) saving resources and expenses. In order to save the transportation cost of the school, all the courses 
are concentrated on the morning or in the afternoon, reducing the number of bus operation. That needs to take the teachers, courses, classes and the time of the class into consideration, making schedule coordinately.

2.2 Elements on Teaching Schedule. Timetable mainly considers the five elements of time, classes, courses, classrooms and teachers. Analyzing those elements is the basis of the arranging model.

1) Time: it includes year, semester, week, day, time in timetable problem. According to the schedule of the school, it only considers the factor of week, when it needs to organize curriculum. There are five schooldays a week and there are ten classes on each school day, each day divided into five periods, each period including 2 classes. The timetable is fixed each semester.

2) Courses: each course has its own number, name, hours and credits, etc., the credit is embodied by the period of the course per week, one credit means one period. When the period of the course per week is odd, it will take its minimum number in teaching course.

3) Classroom: each classroom should have its own number and category (such as ordinary classrooms, multimedia classrooms, computer rooms, etc.), there is only one course held in one classroom during each period, and the type and capacity of the class must meet with the requirement the course at the same time.

4) Class: each class must have its own number and name, and one class can only have one course at the same time.

5) Teacher: each teacher should have their own working number, and a teacher can only teach one course at the same time.

2.3 The Constraints of Timetable Problem. There are a variety of constraints. In order to reduce the complexity of the problem, the arranging constraints are divided into two categories: hard constraints and soft constraints. The former is a necessary principle to be followed in arranging the issue, which is a measure of a practical standard. The latter should be considered, which does not have to be met. However, if you want to have more reasonable results, just meet the requirement of the latter, which is a measure of the merits of the standard program.

Combined with the actual situation of a university, the constraints that need to be considered are: do not conflict constraints, meet the teaching plan constraints and other constraints.

1. Non conflict constraint conditions include:

1) Class does not conflict. The classes which have the same course together can not be arranged the course respectively. For example, the class of 2B1351 and the class of 2B1353 have class together at period 1-2 on Monday, while if the class of 2B1351 will be arranged to have another class at the same time, it will result in class conflict. When having class at the same period, the same class cannot be arranged in different classroom, otherwise it still leads to conflict.

2) Teachers do not conflict. One teacher can not be arranged to have two different courses at the same time. For example, the teacher, Li Dan, can not be scheduled to be on the computer course and database at period 1-2, otherwise, it conflicts.

3) Classroom does not conflict. When arranging courses, two different courses can not be arranged in the same classroom. For example, the class of 1215 has computer class at period 1-2 on Monday, then it can not has not database class meanwhile, otherwise it will produce conflict.

2. the constraints of teaching plan includes:

The total class hours of the course must meet the requirements of the course. Each course can only be arranged in one time and the same classroom. For example, the database program is arranged in class 1215 . If it can't guarantee the teaching plan constraints, it needs to adjust, including adjusting the time and the classroom.

3. Other constraints include:

1) The number of the students must be less than or equal to the classroom capacity. If it exceeds their capacity, such as in computer rooms, multimedia classrooms, voice rooms, and other special classrooms, they can not meet the normal requirement for the students to have class. 
2) According to different course requirements, different types of classroom are arranged. The courses of Multimedia, electronic courses, experimental class, voice class courses, in accordance with the requirements of the curriculum, are arranged in different types of classrooms.

3) When teachers have classes, they can only be arranged in the same campus. For example, the two campus are far away from each other, if a teacher is arranged to have class in the high tech campus at 1-2, it can not arrange the 3-4 period in the Chang an campus, the teacher can not have class on time.

2.4 The Evaluation Criterion of the Result of the Course Scheduling Problem. Timetabling is the optimization combination of time, class, curriculum, classroom and teacher resource, meeting the constraint condition of all kinds of curriculum arrangement as far as possible to improve the teaching quality and teaching management.

1)For students, the best class time is in the morning, they can listen to the class efficiently. Therefore, the course with more credit, curriculum difficulty, or key courses focus on the morning. The rest of the elective course are arranged in the afternoon as far as possible.

2)Try to avoid arranging all day courses. Try to make the students understand and digest as much as possible the course content of the day, that need to keep e as long as one period for their free time. To ensure the smooth progress of the teaching plan, there should not be a full-day class or a free-day class, which requires a careful teaching arrangement.

3)To avoid the far distance of the classroom, which wastes a lot of time for the students when transferring to different classrooms. For example, students have class at period1-2 class in teaching building 1, while period 3-4 is in building in 3, because of the large distance, students will need to spend 10 minutes in coming to the classroom, taking up the student's class time. So in arranging the teaching time, it is appropriate. to arrange students to have class in the same building.

4)When arranging class, the excess of the classroom can not be free. The classroom should be arranged reasonably to ensure that the classroom resources are fully utilized.

5)The work of scientific research projects must be considered when arranging the teaching tasks to the teachers, setting aside 2 days of free time to ensure that the teacher's scientific research and management. Especially for, associate professor and professor senior professional title, they mainly play a leading role in scientific research, so their time should be ensure.

2.5 Curriculum Value Index. For each course is concerned, the value of the curriculum is different. The value of each course should have a standard calculation before arranging. The higher the value of the priority level, it should be considered first. In order to quantify the value, the degree of (Index VI) concept and calculation method of curriculum value index (Value) are given. Define the sections for TN, school classrooms total for RN, the number of the section a certain course requires for $\mathrm{LN}$, the times of this course in a week for RL, the times of this course in a week in the morning class for N1, that of classes in the afternoon for N2. Given that the students take in the knowledge in the morning more than in the afternoon, morning class weight is set to 3 , afternoon classes of weights set to 1 . The formula for calculating the value index is:

$$
V I=\frac{L N}{T N} \times \frac{R N}{R L} \times(3 \times \mathrm{N} 1+\mathrm{N} 2)
$$

If the value of the curriculum is the same, it is arranged in the order of course number.For example, there are 5 sections a day, the total number of classrooms is 200 , the time requirements for the course $\mathrm{A}$ is in the morning (that is, 2 hours), the number of classrooms can be arranged for is 40 . The time requirement for courses $B$ requirements is one period ( 1 hour of the morning), two periods ( 1 section in the afternoon), the number of classroom for courses B is 10.As a result, the value of the course $A$ is:

$$
V I(\mathrm{~A})=\frac{2}{5} \times \frac{200}{40} \times(3 \times 2)=60
$$

The value index of the course $B$ is: 


$$
V I(\mathrm{~B})=\frac{2}{5} \times \frac{200}{10} \times(3 \times 1+1)=32
$$

It is clear that the priority level of the curriculum $\mathrm{A}$ is greater than the priority level of the curriculum B, that is, to give priority to course A when scheduling.

\section{To Establish A Mathematical Model}

The scheduling problem is NP- complete, so it would be difficult to find an efficient global optimization algorithm. Integer programming method is a method of constructing combinatorial optimization problems. When employing Integer programming method to solve the problem, it needs to construct the corresponding constraint condition, the establishment of the equation goal, and then use a appropriate method to solve the problem to solve for the target equations established for the Integer programming.

Based on the scheduling problems, it will build the following mathematical module which mainly describes a strict quantitative relation.

3.1 The Model of Parameters. Based on scheduling problem, it involves in five factors.

Set

1) The set of teachers: $F=\left\{\mathrm{f}_{1}, \mathrm{f}_{2}, \ldots, \mathrm{f}_{\mathrm{M}}\right\}$

2) The set of classrooms: $C=\left\{\mathrm{c}_{1}, \mathrm{c}_{2}, \ldots, \mathrm{c}_{\mathrm{N}}\right\}$

3) The set of course of courses: $L=\left\{1_{1}, 1_{2}, \ldots, 1_{\mathrm{p}}\right\}$

Different teachers having the same course are referred to two classes, and a teacher having the same course for different classes is also referred to two courses, $\mathrm{n}(1)$ : the times of curriculum 1.

4) The set of classes: $R=\left\{\mathrm{r}_{1}, \mathrm{r}_{2}, \ldots, \mathrm{r}_{\mathrm{Q}}\right\}$

5) The set of time: $\quad T=\left\{\mathrm{t}_{1}, \mathrm{t}_{2}, \ldots, \mathrm{t}_{\mathrm{k}}\right\}$

3.2 The Variables of Model. To introduce variables with its values as following:

Teacher $\mathrm{F}$ has a course $\mathrm{L}$ for class $\mathrm{R}$ in time $\mathrm{T}$.

$$
x_{f l c r t}=\left\{\begin{array}{l}
1, \\
0
\end{array}\right.
$$

3.3 The Equation of Constraint. 1. Rigid constraints

$$
f_{1}=\sum c_{n} \varpi_{1 h}
$$

Constraint 1: Each class can not have two different courses at the same period.

$$
R_{1}=\forall c \in C, \forall t \in T \sum_{f \in F} \sum_{r \in R} \sum_{l \in L} f_{\text {flcrt }} \leq 1
$$

Constraint 2: Each teacher can not have two different course at the same period.

$$
R_{2}=\forall f \in F, \forall t \in T \sum_{c \in C} \sum_{r \in R} \sum_{l \in L} f_{\text {flcrt }} \leq 1
$$

Constraint 3: Each classroom can only be arranged one teaching assignment at the same period.

$$
R_{3}=\forall r \in R, \forall t \in T \sum_{f \in F} \sum_{c \in C} \sum_{l \in L} f_{\text {flcrt }} \leq 1
$$

2. Soft constraints

1) Function $\mathrm{f} 1$. The effect of teaching curriculum and class section are related, trying to arrange courses in good sections as much as possible. The teaching effects of section $\mathrm{h}$ shown in set:

$$
R_{3}=\forall r \in R, \forall t \in T \sum_{f \in F} \sum_{c \in C} \sum_{l \in L} f_{\text {flcrt }} \leq 1
$$

2) Function $\mathrm{f} 2$. For the course with more period each week (such as $\geqslant 4$ ), it should be arranged on each other day, with good dispersion of course, it makes a good teaching effect. the teaching effects of Days shown in set: 


$$
f_{1}=\sum c_{n} \varpi_{1 h} \quad f_{2}=\sum c_{n} \varpi_{2 d}
$$

3) Function f3. It's necessary to consider the request of class time and class locations from teachers. Courses teacher has a corresponding relationship with curriculum, and the request of the teacher is cured on the corresponding course. The coefficient of the course's requirement shown in set 6

$$
f_{3}=\sum c_{n} \varpi_{3 j}
$$

3.4 T Target Equation. The course scheduling system can be converted into a linear programming problem, whose objective function can be expressed as follows: the sum of the value of the curriculum reaches the maximum value.

The objective function is defined as:

$$
f(x)=\max \sum V I\left(x_{\text {flcrt }}\right)
$$

The constraint function is defined as:

$$
\text { s.t. } \quad R\left\{\begin{array}{l}
R_{1} \\
R_{2} \\
R_{3}
\end{array}\right.
$$

The objective function The objective formula is to represent the maximum value of curriculum value, that is, the greater the value of the curriculum, the more the better. The constraint function formula is a set representation of the corresponding hard constraints.

\section{Simulated Annealing Algorithm}

4.1 Simulated Annealing Algorithm Model. The simulated annealing algorithm can be divided into three parts as follows: the solution space, the objective function and the initial solution.

The Solution Space. Three conditions of the solution space: the first one is feasible or infeasible solutions; the second one is choosing the initial solution of the constraints; the third one is to generate new solutions, defining a new interpretation of the scope.

Objective Function. Requirements of objective function: firstly, clear optimization objectives, the general optimization into a target and type; secondly, the overall optimization of the problem; finally, easy calculation of the objective function .

Initial Solution. In the implementation of the algorithm, the start point of the program, the selection of initial solution has a great relationship with the final results.

4.2 The Simulated Annealing Algorithm New Generation and Acceptance. Simulated annealing algorithm has asymptotic convergence, the global optimal solution of the optimization algorithm. the following steps of a simulated annealing algorithm and acceptance are as follows:

First step: firstly, to generate the new solutions of the solution space ; secondly, the new solutions is a transform from of the former new solutions. Finally, the transform method determine the scope a new explanation, achieving cooling value which is influenced.

The second step: to calculate and simulate the corresponding difference of the annealing algorithm.

The third step: to determine whether the solution is in line with the requirements

The fourth step: if the new solution meets the requirements, the current solution is replaced by a new solution, and to modify the value of the objective function. The iterative process of a solution is completed at the moment. And then judge again and again to achieve it. If the current solution is invalid, to achieve the judgment on the basis of the original current solution value.

4.3 Simulated Annealing Algorithm. Based on the solution of simulated annealing algorithm, the simulated annealing algorithm with constrained integer programming is investigated, and the flow chart of the algorithm is given, as shown in figure.

$$
\left\{\begin{array}{l}
\frac{\min f(x)}{x \subseteq D} \\
D=\left\{x \mid g_{i}(x) \leq \mathrm{O}(i=1,2, \ldots, s)\right\} \\
x=\left(x_{1}, x_{2}, \ldots, x_{n}\right)(i=1,2, \ldots, n)
\end{array}\right.
$$


In this algorithm, the value of the curriculum is optimized by simulated annealing algorithm, therefore, the value of the exponential function is transferred to the simulated annealing algorithm to obtain the optimal solution by iterative algorithm.

\section{Conclusions}

We give the objective equations of the course arrangement, however, in different teaching environments and with different evaluation of subjective factors, it is difficult to determine the weight of each objective equation. Therefore, the measure for the merits of the timetable is still hard to define. Based on the above, we do not need to find the optimal solution of the problem, but we should seek to the optimum combination to satisfy the constraints $(\mathrm{F}, \mathrm{C}, \mathrm{L}, \mathrm{R}, \mathrm{T})$, The integer programming and simulated annealing algorithm are for better integration and choose one class schedule as optimum. We will make a further study for the algorithms to solve integer programming problems, making the arranging a optimal combination.

\section{References}

[1] Qiang Jin E, A Research on the problem of Course arrangement based on Chaos Genetic Algorithm, in Master's dissertation,Harbin Engineering University,2009.

[2] A.S.Asratian. Investigation of some mathematical model of scheduling theory[D]. Moscow University, 1980.

[3] Yan Dong Yu, A Research on Intelligent Test Paper Generation System based on Genetic Simulated Annealing algorithm, in Master's dissertation,Inner Mongolia University,2011.

[4] C Gotlieb. The Construction of Class - Teacher Time-Tables. 1963.

[5] Research on curriculum arrangement algorithm based on combination of divide-and -conquer with greedy, in Scientific paper,Qingdao Agricultural University,2010

[6] Meng Dexin, Huang Weiwen, Xie Erlian etc.10g [M]. database technology. Oracle Beijing: Tsinghua University press, 2013.

[7] Nick Randolph,David Gardner etc.Visual Studio 2010 Senior programming [M]. Beijing: Tsinghua University press, 2013. 